\title{
Kartagener syndrome
}

\author{
Suat-Jin Lu $\cdot$ Suat W. Loo
}

Received: 26 December 2014/ Accepted: 20 January 2015/Published online: 30 January 2015 (C) SIMI 2015

A 70-year-old woman presented with a 1-week history of dyspnea and cough productive of yellowish sputum. She had previously presented multiple times with similar symptoms. Clinical examination revealed coarse crepitations in both lungs, most severe at the lung bases. A chest radiograph was performed, which showed bronchiectasis in the lower zones of both lungs, more severe on the right side (Fig. 1, white arrow). Both lungs were hyper-inflated with flattening of the diaphragm (Fig. 1, arrowheads). There was dextrocardia. The gastric bubble was seen below the left hemi-diaphragm (Fig. 1, black arrow). The chest radiograph findings were those of Kartagener syndrome. The normal location of the gastric bubble below the left hemidiaphragm excluded situs inversus. Sputum culture grew Pseudomonas aeruginosa. This patient had Kartagener syndrome with infective exacerbation of bronchiectasis. She subsequently showed clinical improvement with antibiotics, supportive care and chest physiotherapy.

Kartagener syndrome, also known as primary ciliary dyskinesia, is an autosomal recessive disorder characterized by a triad of bronchiectasis, dextrocardia/situs inver-

\section{S.-J. Lu ( $\bowtie)$}

Radiology Department, Mount Elizabeth Hospital, 3 Mount

Elizabeth, Singapore 228510, Singapore

e-mail: suat_jin_lu@yahoo.com

S. W. Loo

Department of Oncology, Colchester General Hospital, Turner Road, Colchester, Essex CO4 5JL, UK sus and sinusitis, with defective ciliary motility as the underlying cause $[1,2]$. Situs inversus, not present in this patient, may be present in up to $50 \%$ of patients with Kartagener syndrome [1]. Patients with Kartagener syndrome often have multiple episodes of respiratory tract infection and exacerbation of bronchiectasis due to poor mucociliary clearance. The most useful and convenient diagnostic clue for Kartagener syndrome is the chest radiograph findings of bronchiectasis and dextrocardia/situs inversus.

Bronchiectasis refers to irreversible abnormal dilatation of the bronchi and bronchioles due to repeated infection and inflammation of the airways. This is clinically suspected in patients who present with chronic cough productive of purulent sputum and coarse crackles in the lungs. High resolution computed tomography (HRCT) scan of the chest is currently the most frequently used test to diagnose bronchiectasis and has replaced contrast bronchography [3]. A feature of bronchiectasis on HRCT scan is abnormally dilated bronchi that are bigger than the adjacent bronchial arteries, giving a signet ring appearance [3]. There is also an absence of normal tapering of the bronchial diameter towards the periphery.

In situs inversus totalis, there is a complete transposition of thoracic and abdominal organs. Although situs inversus totalis can easily be overlooked in a busy clinical practice, there are clinical signs that can provide valuable clues for the presence of this condition. For instance, the liver may be felt below the anterior costal margin in the left upper abdomen. The left kidney is, therefore, lower than the right kidney due to the presence of the liver. In a man, the right testis will be lower than the left testis because of a reversal of right and left testicular venous drainage, with the right testicular vein draining into the renal vein instead of the inferior vena cava. 


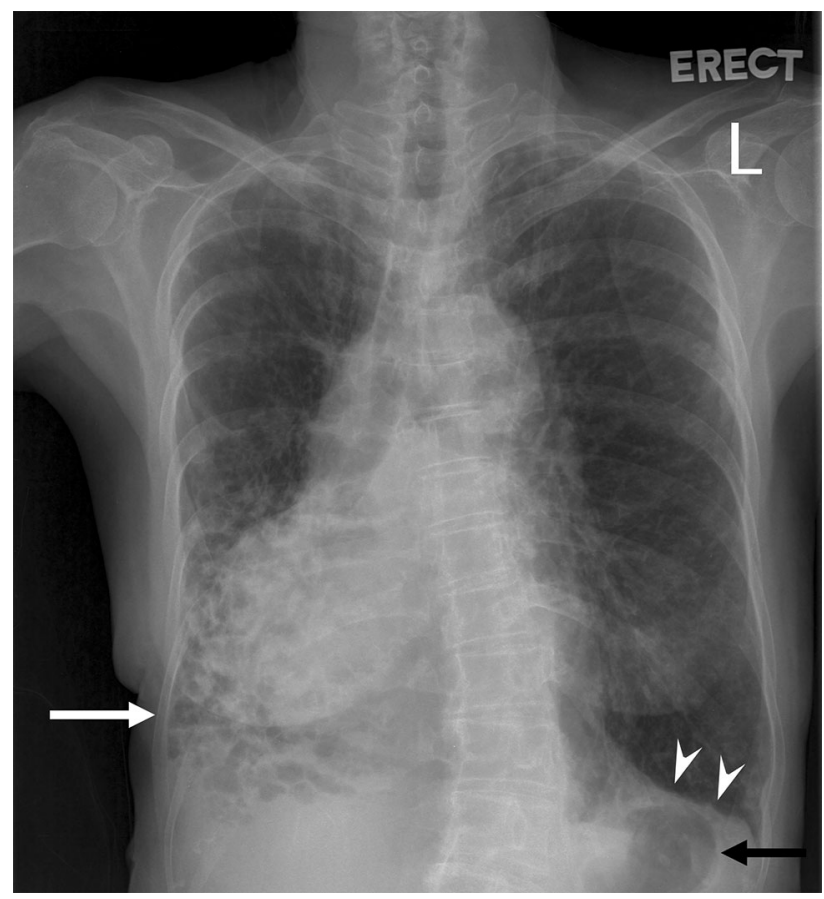

Conflict of interest None.

Fig. 1 Chest radiograph showed bronchiectasis in the lower zones of both lungs, more severe on the right side (white arrow). Both lungs were hyper-inflated with flattening of the diaphragm (arrowheads). There was dextrocardia. The gastric bubble was seen below the left hemi-diaphragm (black arrow)

\section{References}

1. Bush A, Chodhari R, Collins N et al (2007) Primary ciliary dyskinesia: current state of the art. Arch Dis Child 92:1136-1140

2. Rosen MJ (2006) Chronic cough due to bronchiectasis: ACCP evidence-based clinical practice guidelines. Chest 129:122S-131S

3. O'Donnell AE (2008) Bronchiectasis. Chest 134:815-823 